\title{
An accurate shock-capturing finite-difference method to solve the Savage-Hutter equations in avalanche dynamics
}

\author{
Y. G. Tai, ${ }^{1}$ S. Noelle, ${ }^{2}$ J. M. N.T. Gray, ${ }^{3}$ K. Hutter ${ }^{1}$ \\ ${ }^{1}$ Institute for Mechanics, Darmstadt University of Technology, Hochschulstrasse 1, D-64289 Darmstadt, Germany \\ ${ }^{2}$ Institute of Applied Mathematics, Bonn University, Nussallee 12, D-53115 Bonn, Germany \\ ${ }^{3}$ Department of Mathematics, University of Manchester, Manchester M13 9PL, England
}

\begin{abstract}
The Savage-Hutter equations of granular avalanche flows are a hyperbolic system of equations for the distribution of depth and depth-averaged velocity components tangential to the sliding bed. They involve two phenomenological parameters, the internal and the bed friction angles, which together define the earth pressure coefficient which assumes different values depending upon whether the flow is either diverging or contracting. Because of the hyperbolicity of the equations, since velocities may be supercritical, shock waves are often formed in avalanche flows. Numerical schemes solving these free surface flows must cope with smooth as well as non-smooth solutions. In this paper the Savage-Hutter equations in conservative form are solved with a shock-capturing technique, including a front-tracking method. This method can perform for parabolic similarity solutions for which the Lagrangian scheme is excellent, and it is even better in other situations when the latter fails.
\end{abstract}

\section{INTRODUCTION}

The Savage-Hutter equations $(1989,1991)$ are a set of hyperbolic evolution equations that describe the geometry and depth-averaged velocity in finite-mass avalanche flows. The original equations are not written in conservative form and thus can also only reproduce smooth solutions in cases when shock should form. All numerical schemes based on these nonconservative forms generated high oscillations at numerical instabilities in the vicinity where shocks were expected. These were at first damped by adding numerical diffusion but not basing the model on equations in conservative form. This has now been done, and the numerical integration scheme allows the shocks to be identified without the manifestation of spurious oscillations or the formation of instabilities and without the introduction of additional numerical diffusion. As a result, the numerically determined solutions reflect the properties of the model without diffusive contamination.

Shocks are initiated when the avalanche velocity is faster than its characteristic speed and the avalanche front reaches the base of the slope or a solid wall. The shock wave then travels upslope through the avalanche. Many detailed investigations of granular shocks were made by Gray and Hutter (1997), in which the shock waves are considered to be an important feature in the granular flows. The Savage-Hutter equations of granular avalanche flows are a hyperbolic system of equations, such that velocities may be supercritical and shock waves may occur.

To avoid the numerical instability, an artificial viscosity term $\mu \partial^{2} u / \partial x^{2}$ is introduced in the Lagrangian finitedifference method proposed by Savage and Hutter (1989, 1991) for numerical stability, where the artificial viscosity $\mu$ was found to have values of $0.01-0.03$. Such instability is here supposed to be caused by shocks. Guido and Bartelt (unpublished) applied an upwind total variation diminishing
(TVD) difference scheme to solve quasi-Savage-Hutter equations and concluded the numerical stability without using artificial damping with their discretization schemes with respect to the characteristic variables. However, in the quasi-Savage-Hutter and Savage-Hutter equations there are jumps within the characteristics as well as in the characteristic variables. The treatment was not clear in their statement. An alternative is to solve the equations with the conservative variables, the thickness $h$ and the thicknessintegrated moment $m=h u$. In this paper a non-oscillatory central (NOC) shock-capturing numerical scheme (Nessyahu and Tadmor, 1990) is applied to the Savage-Hutter equations $(1989,1991)$ with respect to the conservative variables to describe the shock formation directly, without adding artificial viscosity or other regularizations. Since this numerical scheme is based on a stationary uniform mesh, it is impossible to point out the margin location (the material-free region) without extra treatment. Therefore we combine our NOC scheme with a front-tracking (FT) method, also developed in Tai (2000) and Tai and others (2000). The resulting NOG-FT scheme is able to accurately determine the material-free region.

\section{GOVERNING EQUATIONS}

In the Savage-Hutter theory $(1989,1991)$ the dry cohesionless granular material is assumed to be an incompressible continuum with constant density throughout the entire body. During flow the body behaves as a Mohr-Coulomb plastic material at yield and slides over a rigid basal topography with similar Coulomb-type frictional behaviour. Scaling analysis isolates the physically significant terms in the governing equations and identifies those terms that can be neglected. Finally, depth integration reduces the theory to one spatial dimension. 
The leading-order, dimensionless, depth-integrated mass balance reduces to

$$
\frac{\partial h}{\partial t}+\frac{\partial}{\partial x}(h u)=0
$$

where $h$ is the avalanche thickness and $u$ is the velocity in the downslope direction. The leading-order, dimensionless, depth-integrated momentum balance is

$$
\frac{\partial(h u)}{\partial t}+\frac{\partial}{\partial x}\left(h u^{2}+\beta_{x} h^{2} / 2\right)=h s_{x}
$$

with net driving force

$$
s_{x}=\sin \zeta-\operatorname{sgn}(u) \tan \delta\left(\cos \zeta+\lambda \kappa u^{2}\right),
$$

where $\zeta, \delta$ and $\lambda \kappa$ are local slope inclination angle, basal Coulomb dry-friction angle and local curvature of the chute profile, respectively. The term $\operatorname{sgn}(u)$ comes from the dry Coulomb drag friction, and $\varepsilon \ll 1$ is the aspect ratio of the typical thickness and the length of the avalanche. Note that Equations (1) and (2) are written in conservative form, while in the original Savage-Hutter theory the smoothness assumption yields the momentum-balance equation to the velocity-evolution equation

$$
\frac{\mathrm{d} u}{\mathrm{~d} t}=s_{x}-\beta_{x} \frac{\partial h}{\partial x} .
$$

The factor $\beta_{x}$ is defined as $\beta_{x}=\varepsilon \cos \zeta K_{x}$, and the earth pressure coefficient $K_{x}$ relates the in-plane stresses to the normal stress within the avalanche $K_{x}=p_{x x} / p_{z z}$. The value of $K_{x}$ is given by the Mohr-Coulomb criterion with an ad hoc assumption, depending on whether the avalanche body extends or contracts,

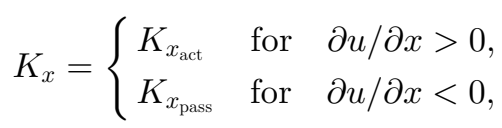

with

$$
K_{x_{\text {act } \mathrm{pass}}}=2\left(1 \pm \sqrt{\frac{1-\cos ^{2} \phi}{\cos ^{2} \delta}}\right) \sec ^{2} \phi-1,
$$

where $\phi$ is the internal friction angle of the granular material.

The original Lagrange finite-difference scheme (Savage and Hutter, 1989) is implemented by the equation system, (1) and (4), in Lagrangian form with primitive variables, i.e. the local thickness of the avalanche $h$, and the downslope velocity $u$. However, the shock-capturing scheme employed here is applied to this equation system in conservative form, (Equations (1) and (2)), with conservative variables, i.e. the thickness $h$ and the thickness-integrated moment $m=h u$.

\section{SHOCK-GAPTURING NUMERIGAL SGHEME}

To describe the shock formation the NOC differencing scheme (Nessyahu and Tadmor, 1990) is here employed, which is similar in form to the Lax-Friedrichs scheme. For completeness, we briefly review the scheme. The fundamental difference between the NOC scheme and standard upwind finitedifference schemes is that the boundaries of the cells at the new time level are the centers of the cells at the old time level (see Fig. 1). In other words, the cell average values $\overline{\mathbf{w}}_{j+1 / 2}^{n+1}$ bounded by $\left[x_{j}, x_{j+1}\right]$ at time level $t^{n+1}$ are computed from the information from the cell average values $\overline{\mathbf{w}}_{j}^{n}$ bounded by $\left[x_{j-1 / 2}, x_{j+1 / 2}\right]$ and $\overline{\mathbf{w}}_{j+1}^{n}$ bounded by $\left[x_{j+1 / 2}, x_{j+3 / 2}\right]$ at time level $t^{n}$ (see Fig. 1, top). This motivates the term "central". For the NOC scheme, the expensive Riemann solvers used in upwind schemes can be completely avoided. The resulting
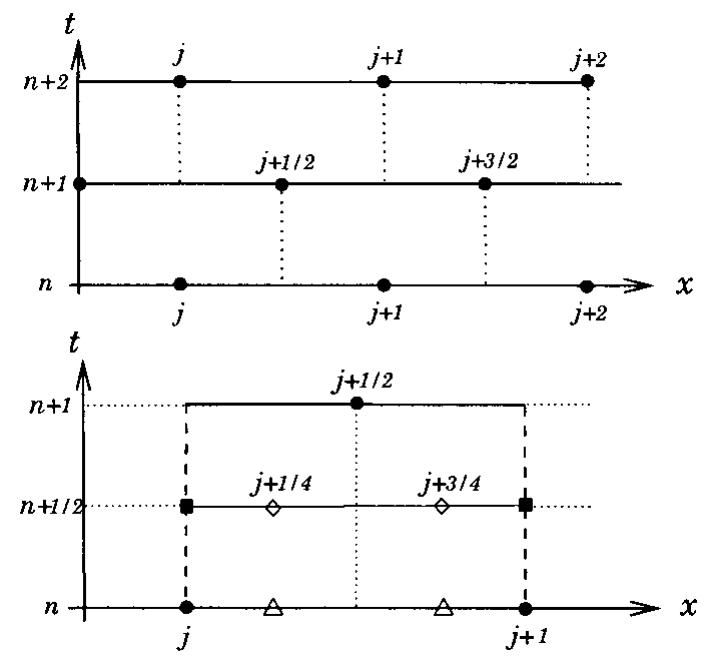

Fig. 1. Diagram of NOC scheme. Top: Gridpoints computed in the NOC method. Bottom: NOC scheme computational diagram, where $\bullet$ indicate the gridpoints at time level $n$ and $n+1$, represent the positions where the fluxes $\boldsymbol{f}$ at time level $n$ $+1 / 2$ are approximated, $\diamond$ are for the source term $s$, and $\triangle$ denote the quarter and three-quarter points meaning (e.g. $\left.\overline{\mathbf{w}}_{j+1 / 4}^{n}, \overline{\mathbf{w}}_{j+3 / 4}^{n}\right)$.

scheme is easy to code, computationally efficient and can be applied to general systems of conservation laws, where the solution of the Riemann problem (i.e. the initial value problem with piecewise constant data) may be complicated or even impossible. For further details and references to recent related work we refer the reader to Tai and others (2000).

For simplicity the Savage-Hutter equations in the form of Equations (1) and (2) are considered with $\boldsymbol{w}=(h, m)^{T}$ as basic variables and written in the form

$$
\boldsymbol{w}_{t}+\boldsymbol{f}_{x}=s
$$

where

$$
\boldsymbol{f}=\left(\begin{array}{c}
m \\
m^{2} / h+\beta_{x} h^{2} / 2
\end{array}\right) \text { and } \quad \boldsymbol{s}=\left(\begin{array}{c}
0 \\
h s_{x}
\end{array}\right) .
$$

Let $\overline{\boldsymbol{w}}_{j}^{n}$ denote the cell average of $\boldsymbol{w}$ at $\left(x_{j}, t^{n}\right)$; integrating the governing equations over the rectangle $\left[x_{j}, x_{j+1}\right] \times$ $\left[t^{n}, t^{n+1}\right]$ gives

$$
\begin{aligned}
\underbrace{\int_{x_{j}}^{x_{j+1}} \boldsymbol{w}^{n+1} \mathrm{~d} x}_{=\overline{\boldsymbol{w}}_{j+1 / 2}^{n+1} \Delta x} & =\int_{x_{j}}^{x_{j+1}} \boldsymbol{w}^{n} \mathrm{~d} x-\int_{t_{n}}^{t_{n+1}}\left(\boldsymbol{f}_{j+1}-\boldsymbol{f}_{j}\right) \mathrm{d} t \\
& +\int_{t_{n}}^{t^{n+1}} \int_{x_{j}}^{x_{j}+1} \boldsymbol{s} \mathrm{d} x \mathrm{~d} t .
\end{aligned}
$$

So, the cell average over $\left[x_{j}, x_{j+1}\right], \overline{\mathbf{w}}_{j+1 / 2}^{n+1}$ can be computed by the following discretized equation:

$$
\begin{gathered}
\overline{\mathbf{w}}_{j+1 / 2}^{n+1}=\frac{1}{2}\left(\overline{\mathbf{w}}_{j+1 / 4}^{n}+\overline{\mathbf{w}}_{j+3 / 4}^{n}\right)-\frac{\Delta t}{\Delta x}\left(\mathbf{f}_{j+1}^{n+1 / 2}-\mathbf{f}_{j}^{n+1 / 2}\right) \\
+\frac{\Delta t}{2}\left(\mathbf{s}_{j+1 / 4}^{n+1 / 2}+\mathbf{s}_{j+3 / 4}^{n+1 / 2}\right)
\end{gathered}
$$

as illustrated in Figure 1 (bottom). The values of $\overline{\mathbf{w}}_{j+1 / 4}^{n}$ and 


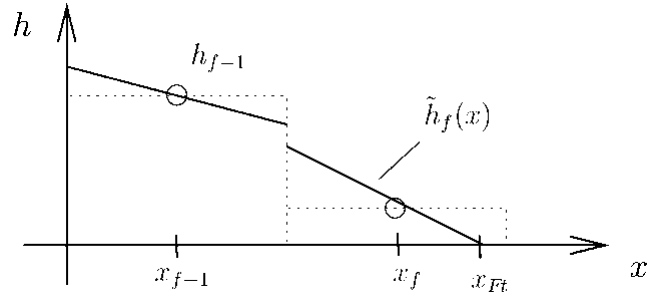

Fig. 2. Cell reconstrution of the depth $\tilde{h}_{f}(x)$ within the frontmargin fth cell. In the front-tracking method a piecewise linear reconstrution $\tilde{h}_{f}(x)$ over the margin cell is well defined, such that the value of the cell average does not change due to the definition of the reconstruction.

$\overline{\mathbf{w}}_{j+3 / 4}^{n}$ are determined by the reconstruction over the $j$ th and $(j+1)$ th cell, i.e.

$$
\begin{aligned}
& \overline{\mathbf{w}}_{j+1 / 4}^{n}=\overline{\mathbf{w}}_{j}^{n}+\frac{1}{4} \overline{\mathbf{w}}_{j}^{\prime}, \\
& \overline{\mathbf{w}}_{j+3 / 4}^{n}=\overline{\mathbf{w}}_{j+1}^{n}-\frac{1}{4} \overline{\mathbf{w}}_{j+1}^{\prime},
\end{aligned}
$$

where $\overline{\mathbf{w}}_{j}^{\prime}$ denotes the cell mean derivative determined by TVD limiter (e.g. Yee, 1989) or the weighted essentially non-oscillatory (WENO) cell reconstruction (Jiang and Shu, 1996).

The transport flux $\mathbf{f}$ is approximated by the physical quantities at $\left(x_{j}, t^{n+1 / 2}\right)$ and $\left(x_{j+1}, t^{n+1 / 2}\right)$, i.e.

$$
\begin{aligned}
\mathbf{f}_{j}^{n+1 / 2} & =\mathbf{f}\left(\overline{\mathbf{w}}_{j}^{n+1 / 2}\right), \\
\overline{\mathbf{w}}_{j}^{n+1 / 2} & =\overline{\mathbf{w}}_{j}^{n}+\frac{\Delta t}{2}(\partial \overline{\mathbf{w}} / \partial t)_{j}^{n} .
\end{aligned}
$$

The integration of the source term represents the volume of $\mathbf{s}$ over $\left[x_{j}, x_{j+1}\right] \times\left[t^{n}, t^{n+1}\right]$, which is approximated by the values at $\left(x_{j+1 / 4}, t^{n+1 / 2}\right)$ and $\left(x_{j+3 / 4}, t^{n+1 / 2}\right)$,

$$
\mathbf{s}_{j+1 / 4}^{n+1 / 2}=\mathbf{s}\left(\overline{\mathbf{w}}_{j+1 / 4}^{n+1 / 2}\right), \quad \mathbf{s}_{j+3 / 4}^{n+1 / 2}=\mathbf{s}\left(\overline{\mathbf{w}}_{\mathbf{j}+\mathbf{3} / \mathbf{4}}^{\mathbf{n}+\mathbf{1} / \mathbf{2}}\right)
$$

and

$$
\begin{aligned}
& \overline{\mathbf{w}}_{j+1 / 4}^{n+1 / 2}=\overline{\mathbf{w}}_{j}^{n}+\frac{\Delta t}{2}(\partial \overline{\mathbf{w}} / \partial t)_{j}^{n}+\frac{1}{4} \overline{\mathbf{w}}_{j}^{\prime}, \\
& \overline{\mathbf{w}}_{j+3 / 4}^{n+1 / 2}=\overline{\mathbf{w}}_{j+1}^{n}+\frac{\Delta t}{2}(\partial \overline{\mathbf{w}} / \partial t)_{j+1}^{n}-\frac{1}{4} \overline{\mathbf{w}}_{j+1}^{\prime} .
\end{aligned}
$$

The temporal derivative $(\partial \overline{\mathbf{w}} / \partial t)_{j}^{n}$ in Equations (12) and (14) is determined by using Equation (7),

$$
\begin{aligned}
(\partial \overline{\boldsymbol{w}} / \partial t)_{j}^{n} & =(\partial \mathbf{f} / \partial x)_{j}^{n}+\mathbf{s}_{j}^{n} \\
& =-\mathbf{A}_{j} \overline{\mathbf{w}}_{j}^{\prime} / \Delta x+\mathbf{s}_{j}^{n},
\end{aligned}
$$

where

$$
\begin{aligned}
& (\partial \mathbf{f} / \partial x)_{j}^{n}=(\partial \mathbf{f} / \partial \mathbf{w})_{j}^{n}(\partial \mathbf{w} / \partial x)_{j}^{n} \\
& \mathbf{A}=\partial \mathbf{f} / \partial \boldsymbol{w}: \text { Jacobean of } \mathbf{f} .
\end{aligned}
$$

To keep the numerical stability and to ensure the non-oscillatory property at a discontinuity the following CFL condition

$$
\frac{\Delta t}{\Delta x}\left|a^{\max }\right|<\frac{1}{2}, \text { for all } j
$$

must hold during computation, where

$$
a^{\max }=\max _{\text {all } j}\left(u_{j}+\sqrt{\beta_{x j} h_{j}}\right)
$$

is the maximum wave speed.

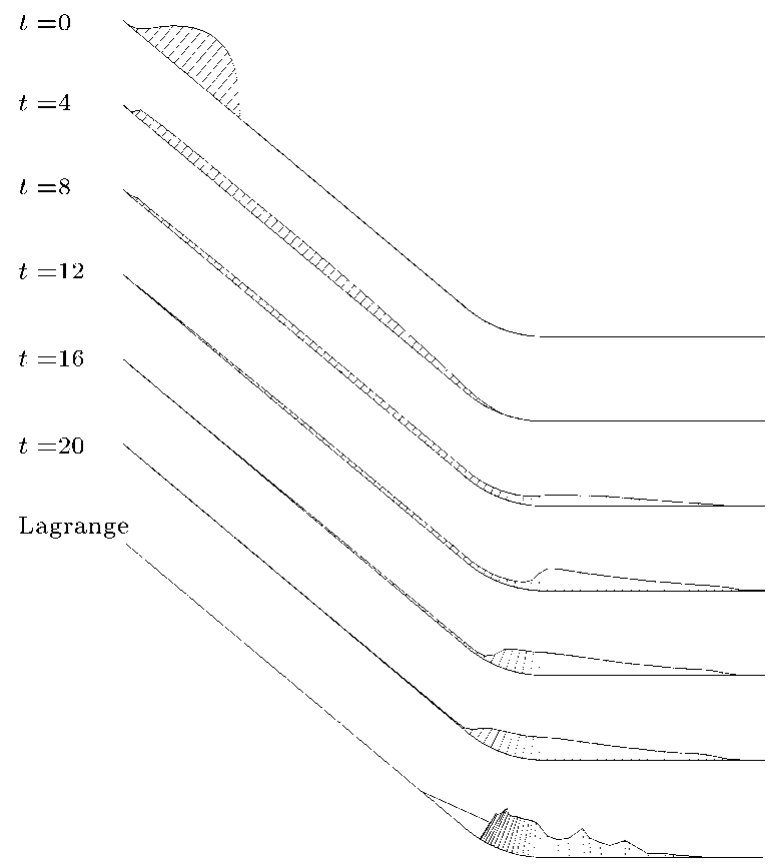

Fig. 3. Process of the avalanche simulated by the shock-capturing NOC method at $t=0,4,8,12,16,20$ dimensionless time units. As the front reaches the run-out zone and comes to rest, the part of the tail accelerates further and the avalanche body contracts. Once the velocity becomes supercritical, a shock wave develops, which moves upward. For the Lagrangian moving-grid method without applying any artificial viscosity, oscillations take place (lowermost panel) when the shock occurs, which may result in an element boundary overtaking the other one and destroying the scheme.

\section{FRONT-TRACKING}

The shock-capturing method discretizes the governing equations on a stationary uniform mesh. The margins may be located between gridpoints, so that it is impossible to point out the margin location without extra treatment. In the front-tracking method used in this paper and developed in Tai and others (2000), the margin cell is reconstructed by a piecewise linear distribution, so that outside the avalanche body there is no material and the cell average does not change due to the reconstruction (e.g. see Fig. 2 for the front margin). It can be easily proven that the margin point fulfils the smoothness assumption (Tai, 2000), and consequently its motion can be described by the following evolution equation

$$
\frac{\mathrm{d} u}{\mathrm{dt}}=s_{x}=\beta_{x} \frac{\partial h}{\partial x}, \quad \text { as } h \rightarrow 0,
$$

which is equivalent to the momentum-balance Equation (4) in the original Savage and Hutter theory. This combination is then called the NOG-FT method. During computation all the numerical fluxes and the source term must be considered if the margin moves into the neighbouring cell.

\section{NUMERIGAL SIMULATIONS}

\section{Upward-moving shock wave}

Shock formations are often observed when the avalanche slides into the run-out horizontal zone. Here the front part comes to rest, while the tail accelerates further and its velocity becomes supercritical. A comparison is made 

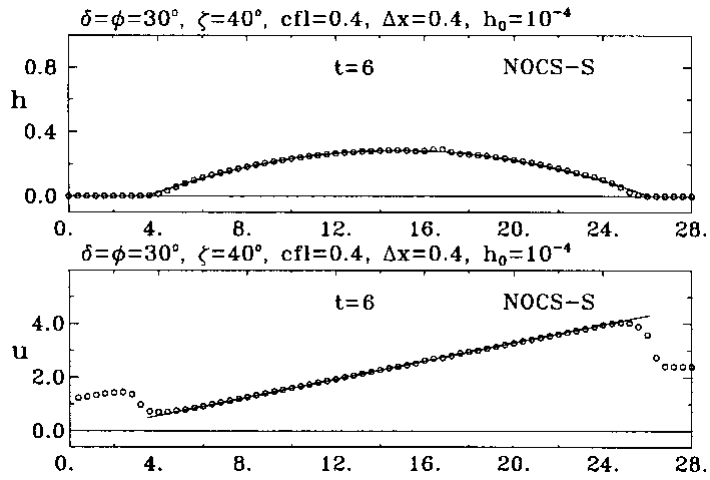

Fig. 4. Depth (upper) and velocity (lower) profiles of the parabolic similarity solution computed by the NOC scheme with the TVD Superbee limiter, where the whole computational domain is divided into 90 cells, the circles denote simulated results and the solid lines represent the exact solution. A thin layer with $h_{0}=10^{-4}$ is added to the whole computational domain.

between the shock-capturing method and the Lagrangian moving grid technique. While such shock formation normally induces numerical instability for the Lagrangian method, the shock-capturing scheme can behave well for such shocks.

The granular material released from a parabolic cap slides down an inclined plane and merges into the run-out horizontal zone. The centre of the cap is initially located at $x=4.0$, and the initial radius and the height are 3.2 and 2.2 dimensionless length units, respectively. The inclination angle of the inclined plane is $40^{\circ}$, and the transition region lies between $x=21.5$ and $x=25.5$. The basal and internal friction angle are both $30^{\circ}$.

Figure 3 illustrates the simulated process as the avalanche slides on the inclined plane into the horizontal runout zone. The avalanche body extends on the inclined plane until the front reaches the horizontal run-out zone. Here the basal friction is enough to bring the front of the granular material to rest, but the part of the rear accelerates further. At this stage, a shock (surge) wave is created $(t=12)$, which moves upward. Such shock waves make the Lagrangian method unstable if no artificial viscosity is applied (see the lowermost panel in Fig. 3).

\section{Parabolic similarity solution}

To test our shock-capturing method for a smooth solution with moving boundaries, the results are compared with the parabolic similarity solution (Savage and Hutter, 1989), which has already been successfully modelled using the Lagrangian technique (Savage and Hutter, 1989, 1991). We refer to Tai (2000) for a generalization of that similarity solution.

Consider a finite mass of granular avalanche sliding on an infinitely long inclined plane at $\zeta=40^{\circ}$, where the internal and basal angles of friction are $\phi=\delta=30^{\circ}$. With a linearly increasing distribution of velocity, $\partial u / \partial x>0$, the avalanche body preserves its parabolic form.

Figure 4 demonstrates the results computed by the NOC scheme without the front-tracking method, where the TVD superbee (e.g. Yee, 1989) limiter is employed and a thin layer $h_{0}=10^{-4}$ is added to the entire computational domain. The results show that the added thin layer does not influence the depth profile very much, if it is sufficiently small, but one cannot determine the margin locations.

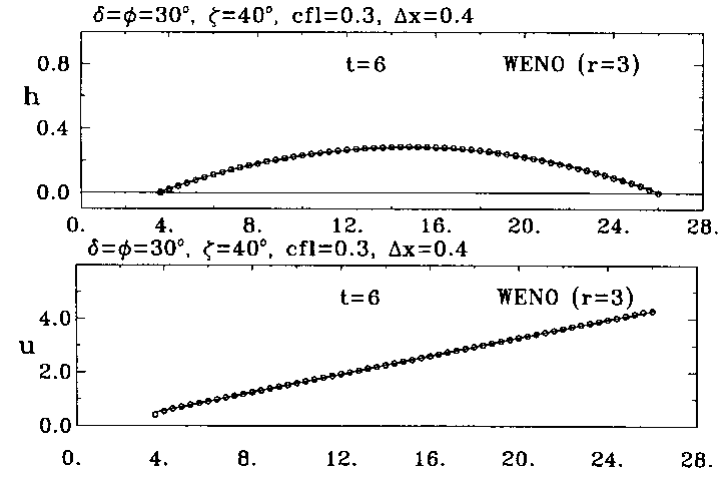

Fig. 5. Depth (upper) and velocity (lower) profiles of the parabolic similarity solution at $t=6$ computed by the $\mathcal{N O C}$ scheme with front-tracking method, where the piecewise quadratic (WENO $r=3$ ) interpolations are implemented. The circles denote simulated results, and the solid lines represent the exact solution. The whole computational domain is divided into 90 cells, and the Courant number is selected to be 0.3.

However, as can be seen in the velocity profiles of Figure 4, there are large velocity gradients around the margins. Since the regions outside the margin are covered by the uniformly added thin layer, there is no contribution from the depth gradient, $\partial h / \partial x$, in the momentum-balance Equation (2). On the other hand, inside the avalanche body there is a permanent contribution from the depth gradient for the momentum balance equation. The velocity in this region will therefore tend to increase differently from that outside the avalanche body, so a jump of velocity develops around the margins. Here the jumps are reproduced by a smeared transition, (see the velocity profiles in Fig. 4). This is why there are deviations of the depth around the margin. Furthermore, the smeared transition of velocity results in several cells with $\partial u / \partial x<0$ around the margins. This violates the presumption $\partial u / \partial x>0$ in the parabolic similarity solution problem. Therefore, the front-tracking method must be introduced.

Near the centre of the avalanche body there is a slight buckling. This is caused by the first-order accurate approximation at the local extremum and is called the clipping phenomenon, which is the weakpoint of the TVD schemes. One way of avoiding this weakness is to apply the higherorder WENO cell reconstruction.

Figure 5 demonstrates the results (circles) from the NOC scheme with the front-tracking method, where a WENO piecewise quadratic interpolation (Jiang and Shu, 1996) is employed. With this method both the parabolic form of the avalanche depth and the margin locations can be well described. The so-called clipping phenomenon is successfully removed. The presumed stress state, $\partial u / \partial x>0$, in the parabolic similarity solution problem is also maintained.

\section{GONGLUSIONS}

The shock-capturing numerical scheme provides an excellent tool to describe shock formation in granular flows with the Savage and Hutter theory. Combining this numerical scheme with the front-tracking method makes it possible to determine the margin locations of the avalanche. Numerical tests of the upward-moving shock wave and a comparison with the parabolic similarity solution confirm the super- 
iority of this combination over previous schemes.Further numerical experiments, including flows for which the basal and internal friction angles are different from each other, are reported in Tai (2000) and Tai and others (2000).

\section{AGKNOWLEDGEMENTS}

This research was supported by the Deutsche Forschungsgemeinschaft projects SFB 298 "Deformation und Versagen bei metallischen und granularen Strukturen" at Darmstadt University of Technology and SFB 256 "Nichtlineare Partielle Differentialgleichungen” at Bonn University.

\section{REFERENCES}

Gray, J. M. N.T. and K. Hutter. 1997. Pattern formation in granular avalanches. Continuum Mech. Thermodyn., 9, 341-345.
Guido, S. and P. Bartelt. Unpublished. Upwinded finite difference schemes for dense snow avalanche modelling. Davos, Eidgenössisches Institute für Schnee- und Lawinenforschung. (Interner Bericht 715.)

Jiang, G. S. and C.W. Shu. 1996. Efficient implementation of weighted ENO schemes. 7. Comput. Phys., 126, 202-228.

Nessyahu, H. and E. Tadmor. 1990. Non-oscillatory central differencing for hyperbolic conservation laws. 7. Comput. Phys., 87, 408-463.

Savage, S. B. and K. Hutter. 1989. The motion of a finite mass of granular material down a rough incline. F. Fluid Mech., 199, 177-215.

Savage, S. B. and K. Hutter. 1991. The dynamics of avalanches of granular materials from initiation to run-out. Part I. Analysis. Acta Mech., 86(14), 201-223.

Tai, Y.-C. 2000. Dynamics of granular avalanches and their simulations with shock-capturing and front-tracking numerical schemes. (Ph.D. thesis, Technische Universität Darmstadt.)

Tai, Y.-C., S. Noelle, J. M. N. T. Gray and K. Hutter. 2000. Shock-capturing and front-tracking methods for granular avalanches. SFB 256 Preprint, Bonn University, Germany.

Yee, H. C. 1989. A class of high-resolution explicit and implicit shock-capturing methods. NASA Tech. Memo. 101088. 